\title{
Bayesian Analysis of a Patient with either Recurrent Metastatic Melanoma or Ipilimumab-Induced Sarcoidosis
}

\author{
Yvonne McNamara ${ }^{1 *}$ and Fergal Kelleher ${ }^{2}$ \\ ${ }^{1}$ Mater Misericordiae University Hospital, Eccles Street, Dublin 7, Ireland \\ ${ }^{2}$ Beacon Hospital, Sandyford, Dublin 18, Ireland
}

Received: 23 June, 2015; Accepted: 21 September, 2015; Published: 05 October, 2015

*Corresponding author: Dr. Yvonne McNamara, Mater Misericordiae University Hospital, Eccles Street, Dublin 7, Ireland, E-mail: yvemcnamara@gmail.com

\begin{abstract}
A patient with metastatic melanoma received treatment with adjuvant Ipilimumab and subsequently developed signs of sarcoidosis 6 weeks post completion of treatment. ${ }^{18} \mathrm{~F}$ FDG PET CT was completed which described FDG avid hilar and mediastinal adenopathy and bilateral FDG avid lung nodules with low-grade bilateral uptake in the lower limbs. Differential diagnosis was either recurrent melanoma or sarcoidosis arising as an immune related adverse event attributable to Ipilimumab. Prior to initiation of antiPD-1 treatment we assessed the merit of undertaking a biopsy to confirm a second relapse of metastatic melanoma. We approached a quantitative appraisal of the clinical dilemma arising in this case using Bayes' theorem and a meta-analysis of published literature of the accuracy of PET detection of recurrent cutaneous melanoma. Partly on this rationale a bronchoscopic biopsy of two lymph nodes was undertaken. Results found abundant histiocytic aggregates focally forming granuloma giving a diagnosis of Ipilimumab induced sarcoidosis. Therefore, positive findings on PET imaging in patients with melanoma treated with Ipilimumab can represent other entities including iatrogenic sequelae from immunotherapy. With the emergence immunotherapy. With the emergence of novel immunotherapy treatment for metastatic melanoma and an increase in the popularity of PET imaging as a modality for diagnosing metastatic disease this article highlights two important issues; the traditional role of biopsies in confirming metastatic disease and immune related adverse events associated with novel therapies.
\end{abstract}

Keywords: Ipilimumab; Melanoma; Ipilimumab induced sarcoidosis; Bayes' theorem; Cancer immunotherapy; Immunerrelated adverse events; FDG PET CT

\section{Introduction}

A 43-year-old male patient had an excision of a nodular melanoma from his anterior abdominal wall in 2007. Breslow's thickness was $1.3 \mathrm{~mm}$. Wide local re-excision and sentinel lymph node biopsy were negative. In 2013, left axillary nodal recurrence was detected. Histologically, melanoma was present in one lymph node, whilst nine further axillary nodes were clear on dissection. Further analysis revealed that the melanoma cells were BRAF V600E positive. LDH was within the normal range. Subsequently he entered a clinical trial of adjuvant Vemurafenib versus placebo. Unblinding revealed him to have been randomised to the placebo study arm. In January 2014, he noted a lump in his left triceps' muscle. A solitary metastasis of $30 \mathrm{~mm}$ in the left triceps was excised with a $1 \mathrm{~mm}$ margin of excision. Ipilimumab was commenced in a pseudo-adjuvant context.

The decision to use Ipilimumab was partly influenced by the patients young age, therefore checkpoint inhibitors, such as Ipilimumab, may have durable complete responses (approximately 20\% for Ipilimumab in metastatic melanoma). These durable complete responses are likely to emerge as the equivalent to a cure. Though drugs such as Vemurafenib or even combined BRAF and MEK inhibitors can improve progression free survival and overall survival, they do not have this effect. It is likely that melanoma is more aggressive in patients with BRAF mutant melanoma progressing on a BRAF inhibitor [1]. Therefore conceptually it is possible that one could deny this young patient the opportunity of curative checkpoint inhibitor treatment with first line BRAF inhibition as post progression he may have a declining performance status. In addition, the kinetics of clinical benefit of Ipilimumab is delayed so the patient could die from progressive disease prior to benefit emerging [1].

A pre surgery MRI brain and 2'-[18F] fluoro-2'-deoxyglucose $\left({ }^{18} \mathrm{~F}\right.$ FDG) PET was otherwise normal. A subsequent ${ }^{18} \mathrm{~F}$ FDG PET computed tomogram 6 weeks post completion of 4 cycles of Ipilimumab described FDG avid hilar and mediastinal adenopathy and bilateral FDG avid lung nodules. SUV ${ }_{\text {max }}$ of the most avid right hilar node was 14.1. There was low-grade uptake in sites of bilateral lower limb subcutaneous oedema. An ultrasound of an area of the lower limb swelling found local areas of subcutaneous oedema. Ultrasound guided fine needle aspiration of an area of low-grade uptake found blood but no malignant cells. In view of the distribution of the likely recurrent melanoma we considered the possibility of this radiology representing sarcoidosis as an immune related adverse reaction secondary to Ipilimumab. Clinically the lower limb findings were consistent with a distribution for erythema nodosum but not classical in clinical appearance. Prior to initiation of anti-PD1 we assessed the merit of undertaking a biopsy to confirm a second melanoma relapse. 


\section{Methods}

Quantitative appraisal of the clinical dilemma arising in this case can be approached using Bayes' theorem [2]:

$$
\frac{p(E / R)}{p(E) p(R / E)+p(e) p(R / e)}=p(E) p(R / E)
$$

$\mathrm{p}(\mathrm{E} / \mathrm{R})$ : probability of the patient having recurrent melanoma after a positive PET $\mathrm{p}(\mathrm{E})$ : probability of the patient having recurrent melanoma before the PET scan

$\mathrm{p}(\mathrm{R} / \mathrm{E})$ : sensitivity of the PET scan

$\mathrm{p}(\mathrm{e})$ : complement of $\mathrm{p}(\mathrm{E})$

$\mathrm{p}(\mathrm{R} / \mathrm{e})$ : false positive rate [2]

In a meta-analysis of published literature of the accuracy of PET detection of recurrent cutaneous melanoma the following values were determined [3-9].

\begin{tabular}{|c|c|c|c|c|}
\hline & $\mathbf{n}$ & Diagnostic value & $\mathbf{\%}$ & $\mathbf{9 5 \%}$ CI \\
TP & 209 & Sensitivity & 96 & $92-98$ \\
FP & 17 & Specificity & 92 & $87-95$ \\
TN & 180 & PPV & 92 & - \\
FN & 9 & NPV & 95 & - \\
\hline
\end{tabular}

TP: True Positives; FP: False Positives; TN: True Negatives; FN: False Negatives; PPV: Positive Predictive Value; NPV: Negative Predictive Value.

\section{Level B and C studies, 6 in total, $n=714$ patients}

We separately determined the likely relapse rate of melanoma post Ipilimumabp(E) to be as follows: Phase II trials give a rate of $30 \%$ for ipilimumab treated patients having disease control at week 12 (CR, PR or SD). Potential sources of error include (i) Ipilimumab was administered in the context of a solitary resected metastasis (ii) there is also a difference between PET scan results weeks 12 and 18 with delayed Ipilimumab tumor kinetics and immune related response criteria of relevance [10]. However the literature is a reasonable approximation. Therefore $p(E)=0.7$

The published literature relevant to these values was assessed.

The accuracy of PET in determining recurrent melanoma: $p$ $(\mathrm{E} / \mathrm{R})=$

$$
\frac{0.7 \times 0.96}{(0.7 \times 0.96)+(0.3 \times 0.023}=0.989
$$

ie; there is a $1.1 \%$ chance of the PET finding representing an entity other than melanoma.

The value $p(E)$ of 0.7 is a theoretical maximum at 18 weeks post initiation of Ipilimumab and is likely far lower. Reasons include relapse at sites other than those described in the PET scan. If even one further reduction of likelihood of relapse is made eg; that resected oligometastatic disease in the pre-Ipilimumab era had a $30 \%$ survival rate then the $p(E)$ value is 0.4 with a $p(E / R)$ of 0.974 and a $2.6 \%$ chance the PET represents disease other than melanoma. As mentioned a long list of factor of intangible quantification will further increase the change of the PET being due to another cause. Partly on this rational an endoscopic biopsy of a station 7 and station 4R lymph nodes was undertaken.

\section{Results}

Endobronchial biopsyat station $4 \mathrm{R}$ lymph node, found abundant histiocytic aggregates focally forming granuloma. There were no melanoma cells in station 7 or station $4 \mathrm{R}$ nodes. In addition to this the serum ACE level was elevated to 90 UECA (NR: 12-68 UECA). The diagnosis therefore was Ipilimumab induced sarcoidosis.

\section{Conclusion}

A positive finding on PET imaging in patients with melanoma treated with Ipilimumab can represent other entities including iatrogenic sequelae from immunotherapy [11]. With the emergence of novel immunotherapy treatment for metastatic melanoma and an increase in the popularity of PET imaging as a modality for diagnosing metastatic disease this article highlights two important issues; the traditional role of biopsies in confirming metastatic disease and immune related adverse events associated with novel therapies [1]. Evaluation of radioisotopes other than $\left[{ }^{18} \mathrm{~F}\right]-\mathrm{FDG}$ including the thymidine analog $3^{\prime}$-deoxy-3'-[ $\left.{ }^{18} \mathrm{~F}\right]$ fluorothymidine $\left[{ }^{18} \mathrm{~F}\right]$ FLT is of interest in this context.

\section{References}

1. Robert C, Ribas A, Wolchok JD, Hodi FS, Hamid O, Kefford R, et al. Anti-progammed-death-receptor-1 treatment with pembrolizumab in Ipilimumab-refractory advanced melanoma: a randomised dosecomparison cohort of a phase 1 trial. The Lancet. 2014; 384: 1109117.

2. Bibb J, Hromas R, Rabinowitz I. A Bayesian approach to a patient with a residual mass after treatment for non-Hodgkin's lymphoma of the thyroid. J Clin Oncol. 2005; 23(34): 8911-3.

3. Danielsen M, Højgaard L, Kjær A, Fischer BM. Positron emission tomography in the follow-up of cutaneous malignant melanoma patients: a systematic review. Am J Nucl Med Mol Imaging. 2013; 4(1):17-28.

4. Koskivuo IO, Seppänen MP, Suominen EA, Minn HR. Whole body positron emission tomography in follow-up of high risk melanoma. Acta Oncol. 2007; 46(5): 685-90.

5. Beasley GM, Parsons C, Broadwater G, Selim MA, Marzban S, Abernethy $\mathrm{AP}$, et al. A multicenter prospective evaluation of the clinical acuity of F-18 FDG-PET/CT in patients with AJCC stage IIIB or IIIC extremety melanoma. Ann Surg. 2012; 256(2): 350-356.

6. Raymond AK, Beasley GM, Broadwater G, Augustine CK, Padussis JC, Turley R, et al. Current trends in regional therapy for melanoma: lessons learned from 225 regional chemotherapy treatments between 1995 and 2010 at a single institute. J Am Coll Surg. 2011; 213(2): 306316.

7. Abbott RA, Acland KM, Harries M, O’Doherty M. The role of positron emission tomography with computed tomography in the follow-up of asymptomatic cutaneous malignant melanoma patients with a high risk of disease recurrence. Melanoma Res. 2011; 21(5): 446-449.

8. Peric B, Zagar I, Novakovic S, Zgajnar J, Hocevar M. Role of serum 
S100B and PET-CT in follow up of patients with cutaneous melanoma. BMC Cancer. 2011; 11: 328.

9. Reinhardt MJ, Joe AY, Jaeger U, Huber A, Matthies A, Bucerius J, et al Diagnostic performance of whole body dual modality 18F-FDG PET/ $\mathrm{CT}$ imaging for $\mathrm{N}$ - and $\mathrm{M}$-staging of malignant melanoma: experience with 250 consecutive patients. J Clin Oncol 2006; 24(7): 1178-1187.

10. Wolchok JD, Hoos A, O’Day S, Weber JS, Hamid O, Lebbé C, et al.
Guidelines for the evaluation of immune therapy activity in solid tumors: immune-related response criteria. Clin Cancer Res. 2009; 15(23): 7412-20.

11. Vogel WV1, Guislain A, Kvistborg P, Schumacher TN, Haanen JB, Blank CU. Ipilimumab-induced sarcoidosis in a patient with metastatic melanoma undergoing complete remission. J Clin Oncol. 2012; 30(2): 